\title{
A genome-wide scan of male sexual orientation
}

\author{
Journal of Human Genetics (2010) 55, 131-132; doi:10.1038/jhg.2009.135; published online 8 January 2010
}

Genetic-epidemiological studies provide some evidence for a genetic component to male homosexuality. ${ }^{1}$ Mustanski et al. ${ }^{2}$ performed the first genome-wide linkage scan for male homosexuality using 403 microsatellite markers. The highest multipoint logarithm of the odds (LOD) score found by Mustanski et al. ${ }^{2}$ was 3.45 near the microsatellite D7S798 on chromosome $7 \mathrm{q} 36$ with approximately equivalent maternal and paternal contributions. As with all studies of complex traits, this result requires confirmation.

Single-nucleotide polymorphism (SNP)based scans for linkage provide significantly greater genomic coverage and information content than do microsatellite assays; ${ }^{3}$ hence, we applied this technology to study a Canadian cohort of homosexual males.
A total of 55 Canadian Caucasian families with two or more homosexual male siblings were studied, ascertained as previously described. ${ }^{4}$ DNA samples were normalized for concentration before labeling and hybridization to the Illumina HumanLinkage-12 BeadChip Infinium array (Illumina, San Diego, CA, USA), which comprises $\sim 6000$ welldefined SNPs spread comparatively evenly across the human genome. The markers on the array exhibit low levels of linkage disequilibrium, and are thus suited for initial genome-wide screens. Genotype calls, generated using BeadStudio (Illumina), were obtained for 112 individuals. Pedigrees were verified using Pedstats, ${ }^{5}$ and data sets checked for unlikely genotypes; suspect sample SNP data points were removed from subsequent analyses. Nonparametric linkage analyses were carried out using MERLIN (http:// www.sph.umich.edu/csg/abecasis/Merlin/ index.html). ${ }^{6}$

The results of the genome-wide linkage scan are shown in Figure 1.

A LOD score peak of 2.86 was obtained on chromosome 14 for SNP rs760335 $(98.8 \mathrm{cM}$, position 93884697 ; genome build 36 ). The adjacent SNP loci are rs733559 $(96.8 \mathrm{cM}$; $92809308, \mathrm{LOD}=2.08)$ and $\mathrm{rs742893}(99.7$ cM; $94222866, \mathrm{LOD}=1.74)$. Modeling of marker-marker linkage disequilibrium in the data set $\left(r^{2}>0.1\right)$ did not significantly alter the magnitude of LOD scores obtained (rs760335, maximum LOD=2.47). However, when we analyzed 1000 simulated data sets of pedigree genotypes generated by MERLIN, a LOD score of $\geqslant 2.47$ was obtained in 256 of the 1000 simulations, generating an empirical

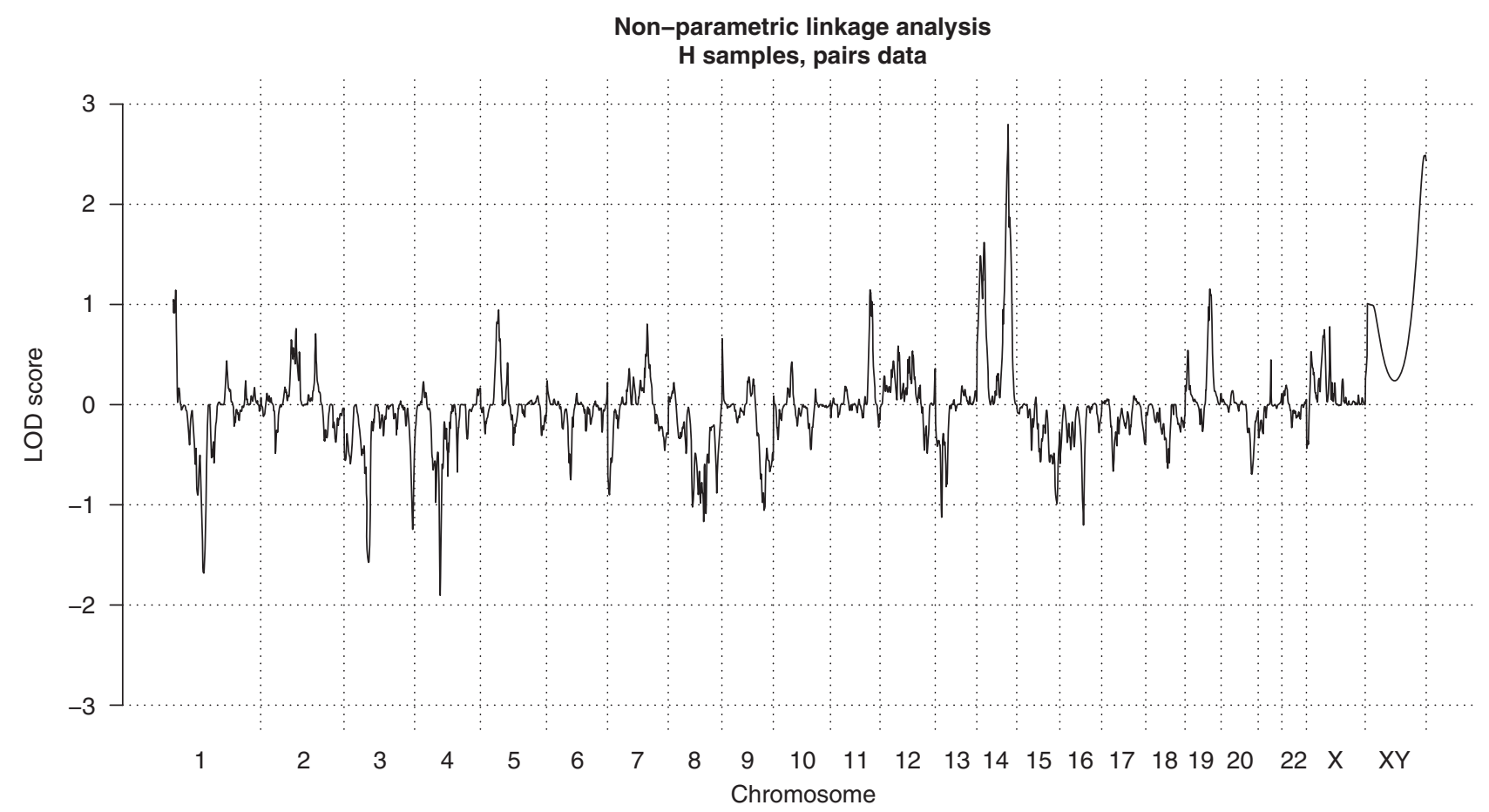

Figure 1 Genome-wide LOD scores for linkage across all chromosomes. 
$P$-value for our linkage peak of 0.256 . There was no evidence for linkage at or around $7 \mathrm{q} 32(\mathrm{LOD}=-0.2)$.

Therefore, in summary, although we could find no evidence for linkage at $7 \mathrm{q} 32$ as found by Mustanski et al. ${ }^{2}$ and as the LOD scores we obtained failed to reach genome-wide significance as defined by Lander and Kruglyak, ${ }^{7}$ the studies combined provide information regarding the magnitude of effects likely to be attributable to male homosexuality genes if they do indeed exist. Differences in the two studies may lie in genetic and population heterogeneity, but it is more probable that genes involved in male homosexuality exert more modest effects than detectable by linkage. ${ }^{8}$ More powerful genetic studies will be required to find any relevant genes. Parent-of-origin effects in male homosexuality have been documented, ${ }^{2}$ and thus consideration of epigenetic mechanisms are also strongly warranted. ${ }^{9}$
Sreeram V Ramagopalan ${ }^{1,2}$, David A Dyment ${ }^{1,2}$, Lahiru Handunnetthi ${ }^{1,2}$, George P Rice ${ }^{3}$ and George C Ebers ${ }^{1,2}$

${ }^{1}$ Wellcome Trust Centre for Human Genetics, University of Oxford, Oxford, UK; ${ }^{2}$ Department of Clinical Neurology, The John Radcliffe Hospital, University of Oxford, Oxford, UK and 3 Department of Clinical Neurological Sciences, University of Western Ontario, London, Ontario, Canada E-mail: george.ebers@clneuro.ox.ac.uk

1 Bailey, J. M., Pillard, R. C., Dawood, K., Miller, M. B., Farrer, L. A., Trivedi, S. et al. A family history study of male sexual orientation using three independent samples. Behav. Genet. 29, 79-86 (1999).

2 Mustanski, B. S., Dupree, M. G., Nievergelt, C. M., Bocklandt, S., Schork, N. J. \& Hamer, D. H. A genomewide scan of male sexual orientation. Hum. Genet. 116, 272-278 (2005).
3 Middleton, F. A., Pato, M. T., Gentile, K. L., Morley, C. P., Zhao, X., Eisener, A. F. et al. Genomewide linkage analysis of bipolar disorder by use of a high-density single-nucleotide-polymorphism (SNP) genotyping assay: a comparison with microsatellite marker assays and finding of significant linkage to chromosome $6 \mathrm{q} 22$. Am. J. Hum. Genet. 74, 886-897 (2004).

4 Rice, G., Anderson, C., Risch, N. \& Ebers, G. Male homosexuality: absence of linkage to microsatellite markers at Xq28. Science 284, 665-667 (1999).

5 Wigginton, J. E. \& Abecasis, G. R. PEDSTATS: descriptive statistics, graphics and quality assessment for gene mapping data. Bioinformatics 21, 3445-3447 (2005).

6 Abecasis, G. R., Cherny, S. S., Cookson, W. O. \& Cardon, L. R. Merlin—rapid analysis of dense genetic maps using sparse gene flow trees. Nat. Genet. 30, 97-101 (2002).

7 Lander, E. \& Kruglyak, L. Genetic dissection of complex traits: guidelines for interpreting and reporting linkage results. Nat. Genet. 11, 241-247 (1995).

8 Risch, N. \& Merikangas, K. The future of genetic studies of complex human diseases. Science 273, 1516-1517 (1996).

9 Ramagopalan, S. V., Dyment, D. A. \& Ebers, G. C. Genetic epidemiology: the use of old and new tools for multiple sclerosis. Trends Neurosci. 31, 645-652 (2008). 